\title{
Improving the odds of success in antitumoral drug development using scoring approaches towards heterocyclic scaffolds
}

\author{
GEORGE NICOLAE DANIEL ION ${ }^{1}$, OCTAVIAN TUDOREL OLARU ${ }^{1}$, GEORGIANA NITULESCU ${ }^{1}$, \\ IULIA IOANA OLARU ${ }^{1}$, ARISTIDIS TSATSAKIS ${ }^{2,3}$, TATIANA I. BURYKINA ${ }^{3}$, \\ DEMETRIOS A. SPANDIDOS ${ }^{4}$ and GEORGE MIHAI NITULESCU ${ }^{1}$ \\ ${ }^{1}$ Faculty of Pharmacy, 'Carol Davila' University of Medicine and Pharmacy, 020956 Bucharest, Romania; \\ ${ }^{2}$ Department of Forensic Sciences and Toxicology, Faculty of Medicine, University of Crete, 71003 Heraklion, \\ Greece; ${ }^{3}$ Department of Analytical and Forensic Medical Toxicology, Sechenov Medical University, 119991 Moscow, \\ Russia; ${ }^{4}$ Laboratory of Clinical Virology, School of Medicine, University of Crete, 71003 Heraklion, Greece
}

Received April 2, 2020; Accepted June 5, 2020

DOI: $10.3892 /$ or.2020.7636

\begin{abstract}
One of the most commonly discussed topics in the field of drug discovery is the continuous search for anticancer therapies, in which small-molecule development plays an important role. Although a number of techniques have been established over the past decades, one of the main methods for drug discovery and development is still represented by rational, ligand-based drug design. However, the success rate of this method could be higher if not affected by cognitive bias, which renders many potential druggable scaffolds and structures overlooked. The present study aimed to counter this bias by presenting an objective overview of the most important heterocyclic structures in the development of anti-proliferative drugs. As such, the present study analyzed data for 91,438 compounds extracted from the Developmental Therapeutics Program (DTP) database provided by the National Cancer Institute. Growth inhibition data from these compounds tested on a panel of 60 cancer cell lines representing various tissue types (NCI-60 panel) was statistically interpreted using 6 generated scores assessing activity, selectivity, growth inhibition efficacy and potency of different structural scaffolds, Bemis-Murcko skeletons,
\end{abstract}

Correspondence to: Dr Georgiana Nitulescu, Faculty of Pharmacy, 'Carol Davila' University of Medicine and Pharmacy, 6 Traian Vuia, 020956 Bucharest, Romania

E-mail: georgiana.nitulescu88@gmail.com; georgiana.nitulescu@ umfcd.ro

Abbreviations: CAS Registry, Chemical Abstracts Service Registry; $\mathrm{GI}_{50}$, half maximal growth inhibition; IQR, interquartile range; $\mathrm{LC}_{50}$, half maximal lethal concentration; NCI, National Cancer Institute; NSC Number, Cancer Chemotherapy National Service Center number; TGI, total growth inhibition

Key words: NCI-60 cell line panel, Bemis-Murcko scaffolds, privileged structures, screening oncology compounds, drug design, anti-proliferative activity, growth inhibition, cancer cell line chemical features and structures common among the analyzed compounds. Of the most commonly used rings, the most prominent anti-proliferative effects were produced by quinoline, tetrahydropyran, benzimidazole and pyrazole, while overall, the optimal results were produced by complex ring structures that originate from natural compounds. These results highlight the impact of certain ring structures on the anti-proliferative effects in drug design. In addition, considering that medicinal chemists usually focus their research on simpler scaffolds the majority of the time with no significant pay-off, the present study indicates several unused complex scaffolds that could be exploited when designing anticancer therapies for optimal results in the fight against cancer.

\section{Introduction}

The design and development of novel therapeutic small molecules is a major research field and a main focus of this research is the identification of more effective anticancer solutions (1). A number of powerful and diverse array of methods have been developed to improve the success of drug design based on the three-dimensional structure of the pharmacological target (2-5); however, numerous researchers are still successfully using the ligand-based strategies. These methods are based on the simple principle that similar structures will produce the same biological effect and have the advantage of a lower risk of failure $(6,7)$. A closer analysis of the drug design process has revealed that medicinal chemistry specialists rely on their intuition when deciding the direction of the research, and often, they are affected by cognitive biases $(8,9)$. One of the most important is the so-called confirmation bias. This represents the tendency of researchers to consider the data that support their hypotheses to a greater extent and not to actively search for evidence that would contradict their hypothesis (9).

The confirmation bias can be easily observed by reading most of the medicinal chemistry articles in which researchers are supporting their focus on a particular type of structure by presenting various similar compounds sharing the targeted biological effect. For example, Sharma et al considered the thiazole ring as an essential core scaffold for anticancer drugs 
by presenting clinically proved drugs, such as dasatinib, dabrafenib, or ixabepilone (10). Indole is another heterocycle that is regarded as useful in the target-based design of anticancer agents based on similar reasoning (11). Natural scaffolds, such as the flavonoid core structure, are extensively used in the design of novel cancer drugs $(12,13)$. Ismail et al argued that pyrazolo[3,4-d]pyrimidine derivatives have a good chance to target protein kinases and to be developed as anticancer agents (14). Our own research group has focused on a particular chemophore, the pyrazole ring, as a scaffold for the design of anticancer agents (15-18).

The objective of the present study was to identify an unbiased quantitative method which may be used to measure the usefulness of the most important heterocyclic structures in the development of anti-proliferative drugs. The scope of the present study was to help chemists focus on the chemical structure with better potential, as well as to understand their bias towards a particular scaffold.

Cancer cell lines serve as a major model for antineoplastic drug discovery and development. The National Cancer Institute (NCI) established a systematic screening program by assembling a panel of 60 cancer cell lines (NCI-60 panel) from multiple tumor types (brain, blood and bone marrow, breast, colon, kidney, lung, ovary, prostate and skin) $(19,20)$. Over the past decades, a large number of compounds have been tested on the NCI- 60 panel and several cytotoxic agents have emerged as first-line treatment options for a number of tumor types (21).

A compound is first tested at a single concentration and then, if found active, it is tested at five different concentrations with 48 -h drug exposure and $50 \%$ growth inhibition $\left(\mathrm{GI}_{50}\right)$, total growth inhibition (TGI), and 50\% lethal concentration $\left(\mathrm{LC}_{50}\right)$ are computed (22). Data analysis tools, such as COMPARE use these outputs on all 60 cancer cell lines to create a fingerprint profile that allows classification and can predict the mechanisms of action $(23,24)$. The fingerprint of cellular response in the NCI-60 assay can be used to determine similar prototype compounds, the usefulness of this data mining approach being demonstrated in various studies $(25,26)$.

\section{Materials and methods}

Creation and preparation of datasets. Two sets of data were collected freely from the DTP website (https://dtp.cancer. gov/databases_tools/default.htm) representing one-dose screening values and five-dose screening data. The number reported in the one-dose data set is each cell line growth percentage (GI\%) following $48 \mathrm{~h}$ of exposure to $10^{-5} \mathrm{M}$ solution of drug, relative to the no-drug control, for a specific cell line. A GI\% value $<100$ and $>0$ indicates a growth inhibition and a value $<0$ represents a lethal effect of the tested compound. The 5-dose screening data set contains the negative $\log 10$ of the $50 \%$ growth inhibitory concentration expressed as molar concentration $\left(\mathrm{pGI}_{50}\right)$ for each tested compound.

Bemis-Murcko scaffold analysis. Bemis-Murcko skeletons represent the molecular frameworks resulting after the removal of the side-chain atoms and atom labels. All bond types are transformed into single ones (10). The analysis was performed using DataWarrior 5.2.0 (http://www.openmolecules.org/data- warrior/) to generate the Bemis-Murcko scaffolds representing the cyclic frameworks incorporating only the rings and the chains connecting them. Bemis-Murcko scaffolds underline the importance of molecular topology and have been proven to be useful in several drug design studies (11-15).

Plain ring analysis. DataWarrior 5.2.0 software was used to identify all ring systems in each compound without substituents. The double bonded heteroatoms connected directly to the ring system were taken into consideration. Both procedures for Bemis-Murcko scaffold and plain ring using sunitinib the Cancer Chemotherapy National Service Center number [(NSC Number) 750690] as an example are illustrated in Fig. 1.

Scoring methods. The one-dose data set matrix was analyzed in order to assess the performance of each chemical feature on generating potent anti-proliferative candidates. The average $\left(\mathrm{A}_{1 \mathrm{D}}\right)$ of all GI\% values registered for a specific scaffold was calculated as an indicator of the anti-proliferative potency of that chemical feature as follows:

$$
A_{1 \mathrm{D}}=\frac{\sum \mathrm{GI} \%}{\text { No. of cell lines }}
$$

For each scaffold, a performance score $\left(\mathrm{P}_{1 \mathrm{D}}\right)$ was defined as the number of GI\% values under the $50 \%$ value threshold reported to the total number of GI\% values recorded for that scaffold. The corresponding mathematical formula is as follows:

$$
P_{1 D}=100 \times \frac{\text { No. of cell lines GI\% } \leq 50}{\text { No. of cell lines }}
$$

The scoring formula is similar to the measure of incidence in epidemiology (27) considering an observed event an anti-proliferative effect $>50 \%$.

A third score was implemented to assess the selectivity of the anti-proliferative effect based on the number of outliers. Considering that each compound is tested on a panel of 60 cancer cell lines, a vector of data results. The lower outliers were identified as any GI\% data below the Tukey's lower boundary, a threshold (TLi) based on the interquartile range (IQR) and the first quartile (Q1) using the following formula:

$$
\begin{gathered}
\mathrm{IQR}=\mathrm{Q} 3-\mathrm{Q} 1 \\
\mathrm{TLi}=\mathrm{Q} 1-1.5 \times \mathrm{IQR}
\end{gathered}
$$

where 'I' represents the compound number, Q1 and Q3 represent first and third quartiles in the data vector of each compound, while IQR represents the difference between Q3 and Q1. The presence of a low outlier indicates that the corresponding cell is specifically sensitive to the tested compound.

The selectivity score $\left(\mathrm{O}_{1 \mathrm{D}}\right)$ was calculated as the number of $\mathrm{GI} \%$ values below the TLi value reported to the total number of GI\% values recorded for each scaffold. The corresponding mathematical formula is as follows:

$$
\mathrm{O}_{1 \mathrm{D}}=100 \times \frac{\text { No. of cell lines GI\% }<\mathrm{TLi}}{\text { No. of cell lines }}
$$

The same formulas were adjusted to calculate similar scores using the pGI data. In the case of the performance score 


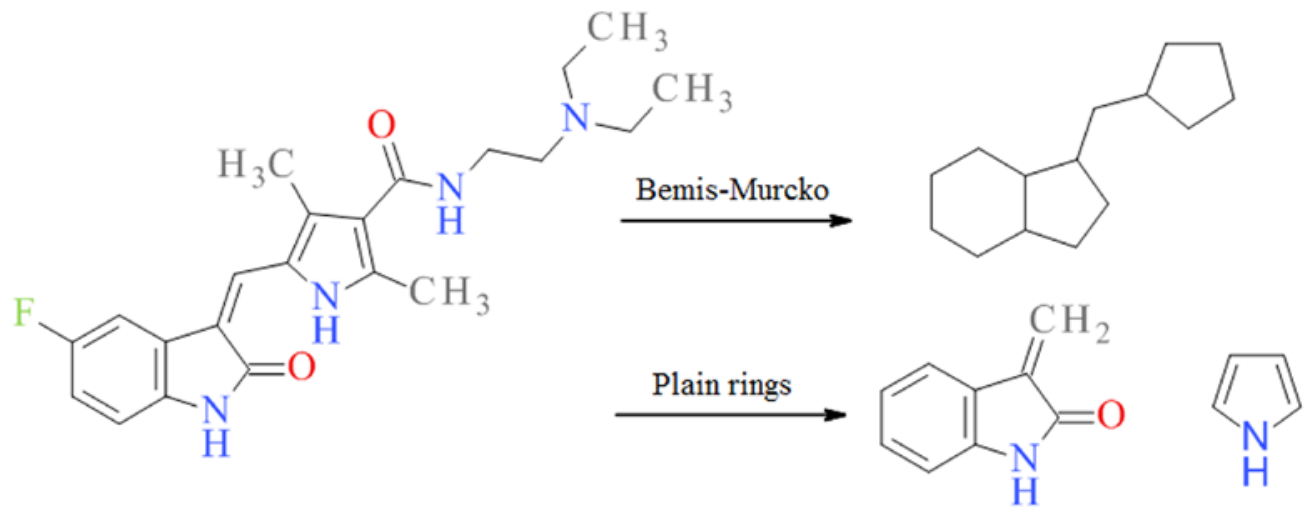

Figure 1. Example of the scaffold generation methods using sunitinib.

$\left(\mathrm{P}_{\mathrm{pGI}}\right)$, the counted values where those $>5$ and in the case of the selectivity score, the counted values were those above the Ti fence.

$$
\begin{gathered}
A_{\mathrm{pGI}}=\frac{\sum \mathrm{pGI}}{\text { No. of cell lines }} \\
\mathrm{P}_{\mathrm{pGI}}=100 \times \frac{\text { No. of cell lines pGI }>5}{\text { No. of cell lines }} \\
\mathrm{O}_{\mathrm{pGI}}=100 \times \frac{\text { No. cell lines pGI } \geq \mathrm{TUi}}{\text { No. of cell lines }}
\end{gathered}
$$

where TUi represents the upper boundary for outlier identification and it is calculated using the following formula:

$$
\mathrm{TUi}=\mathrm{Q} 3+1.5 \times \mathrm{IQR}
$$

In order to reduce false high-positive values caused by a low number of tested cells, all the scores were analyzed only if the total number of data points registered for a specific structure was $>500$.

\section{Results}

Creation of data sets. A total of 284,176 structures were downloaded from the DTP website and were manually curated by eliminating 119 erroneous cases. From this set, a total of 192,619 structures were eliminated as no $\mathrm{GI} \%$ or $\mathrm{pGI}_{50}$ value were associated with the corresponding NSC code. The set of compounds with GI\% values resulted after the analysis of the one-dose set and represents 45,020 unique substances. The compounds with no associated structure were eliminated, yielding a final set of 44,960 compounds (set $\mathrm{GI}_{1 \mathrm{D}}$ ). The $\mathrm{pGI}_{50}$ set contains 52,769 compounds. The compounds with $\mathrm{pGI}_{50}$ values expressed as $\mu \mathrm{g} / \mathrm{ml}$ were eliminated, resulting in a final set of 51,968 compounds (set PGI). The third set (AL) consists of the union of the 2 sets.

$$
\mathrm{AL}=\mathrm{GI}_{1 \mathrm{D}} \cup \mathrm{PGI}
$$

The AL set consists of 91,438 unique compounds characterized by an NSC code, a chemical structure, and at least one biological endpoint (GI\%, pGI, or both).

Bemis-Murcko scaffolds. The structures of the compounds from all 3 data sets were transformed into their Bemis-Murcko skeleton using the procedure described in the methods section. The transformation of all the 91,438 structures in the AL set resulted in 11,763 distinct Bemis-Murcko scaffolds. For a number of 4,106 compounds representing $4.49 \%$ of the set, the results were blank as they did not contain any ring structures.

Despite the high diversity of scaffolds, only 86 of these appear with a frequency above the $0.1 \%$ threshold. The distribution of the scaffolds in all 91,438 structures follows the power law $\left(\mathrm{R}^{2}=0.961\right)$ with the frequency of occurrence for the i-ranked scaffold obeying the following formula:

$$
F(i)=7.066 \times i^{-0.972}
$$

The same type of distribution was observed in a study on the distribution of Bemis-Murcko scaffolds in the organic subset Chemical Abstracts Service Registry (CAS Registry) (28). There are two main reasons for such a distribution: Popularity or performance; however, the distribution alone is not sufficient to understand which of these factors causes it (29). In order to provide an answer to this question, the performance and selectivity scores were implemented.

The top-ranking 10 scaffolds are presented in Table I along with their frequency and corresponding scores $A_{1 D}, A_{p G I}, P_{1 D}$, $\mathrm{P}_{\mathrm{pGI}}, \mathrm{O}_{1 \mathrm{D}}$ and $\mathrm{O}_{\mathrm{pGI}}$.

The results clearly demonstrate that the most popular Bemis-Murcko scaffolds provide little guarantee that the compounds containing them will determine sufficient anti-proliferative effects. The BM10 scaffold presents the optimal scores amongst the top 10 used ones. The scores registered for the non-cyclic compounds are better than those of some BM scaffolds, indicating that the presence of a cyclic structure is not essential.

For all the compounds in the PGI set, the Bemis-Murcko analysis returned 9,540 scaffolds. For each scaffold, the aforementioned scores were calculated. The values were analyzed only if the total number of data points used was $>500$. A total of 662 scaffolds met this criterion. Analyzing the $\mathrm{A}_{\mathrm{pGl}}$ values, only 142 scaffolds $(21.45 \%$ ) had values $>5$, and only 28 scaffolds (4.23\%) presented $\mathrm{A}_{\mathrm{pGI}}$ values $>6$. Of these 28 scaffolds, apart from one two-ringed skeleton, all had between 3 to 7 rings, and at least 14 carbon atoms. Similarly, based on the $\mathrm{P}_{\mathrm{pGI}}$ values, only 121 of the scaffolds produced at least a $50 \%$ growth inhibition in at least $50 \%$ of the tested 
Table I. Most commonly used Bemis-Murcko scaffolds and their anti-proliferative performance scores.

\begin{tabular}{|c|c|c|c|c|c|c|c|c|}
\hline Code & Scaffold & Freq (\%) & $A_{1 D}$ & $\mathrm{~A}_{\mathrm{pGI}}$ & $P_{1 D}$ & $\mathrm{P}_{\mathrm{pGI}}$ & $\mathrm{O}_{1 \mathrm{D}}$ & $\mathrm{O}_{\mathrm{pGII}}$ \\
\hline BM01 & & 12.53 & 100.08 & 4.39 & 1.85 & 16.45 & 2.40 & 3.95 \\
\hline BM02 & & 2.96 & 94.44 & 4.52 & 5.31 & 23.50 & 2.79 & 4.29 \\
\hline BM03 & & 2.80 & 95.56 & 4.65 & 4.28 & 23.76 & 2.63 & 4.07 \\
\hline BM04 & & 2.68 & 99.03 & 4.32 & 2.52 & 12.95 & 2.56 & 3.73 \\
\hline BM05 & & 2.35 & 100.37 & 4.29 & 1.92 & 14.66 & 2.37 & 3.16 \\
\hline BM06 & & 2.08 & 95.07 & 4.57 & 5.28 & 25.08 & 2.64 & 3.62 \\
\hline BM07 & & 1.56 & 92.82 & 4.59 & 6.43 & 24.78 & 2.66 & 4.27 \\
\hline BM08 & & 1.38 & 98.92 & 4.29 & 2.85 & 13.18 & 2.82 & 3.85 \\
\hline BM09 & & 1.30 & 98.13 & 4.40 & 3.35 & 15.13 & 2.62 & 3.77 \\
\hline BM10 & & 1.14 & 78.17 & 4.97 & 18.21 & 43.09 & 2.53 & 3.98 \\
\hline $\begin{array}{l}\text { No } \\
\text { ring }\end{array}$ & - & 4.49 & 100.39 & 4.35 & 2.05 & 16.06 & 2.28 & 3.76 \\
\hline
\end{tabular}

$\mathrm{A}_{1 \mathrm{D}}$, average of GI\% values; $\mathrm{P}_{1 \mathrm{D}}$, number of GI\% values the $<50 \%$ value threshold; $\mathrm{O}_{1 \mathrm{D}}$, number of GI\% lower boundary outliers; $\mathrm{A}_{\mathrm{pGI}}$, average of $\mathrm{pGI}$ values; $\mathrm{P}_{\mathrm{pGI}}$, number of $\mathrm{pGI}$ values the $>5$ value threshold; $\mathrm{O}_{\mathrm{pGI}}$, number of $\mathrm{pGI}$ upper boundary outliers.

cells $\left(\mathrm{P}_{\mathrm{pGI}}>50\right)$. Taking both scores into account, the optimal Bemis-Murcko skeletons are presented in Fig. 2.

The results indicated that the most successful Bemis-Murcko scaffolds had a very large complexity, which explains their limited use in drug design. It seems that the simple chemical structures are preferred, even if their potential to generate good leads is weak.

Plain ring global analysis. The analysis of all of the 91,438 structures in the AL set resulted in 10,074 distinct ring scaffolds. A total of 30,577 compounds (33.44\%) had 1 ring, $31,705(34.67 \%)$ contained 2 plain rings in their structure and $18.47 \%$ contained 3 rings. Considering the frequency of appearance, the first ranked ring was benzene, which had a very high recurrence being present in $45.50 \%$ of the whole AL set, while the second-ranked ring (pyridine) had a frequency of $3.18 \%$.

Only 73 ring scaffolds registered a frequency $>0.1 \%$. Based on the number of ring closures in each scaffold, the 1-ring scaffolds appear in $70.33 \%$ of compounds, followed by the 2-ring scaffolds (16.82\%) and the 3-ring ones (5.94\%). 

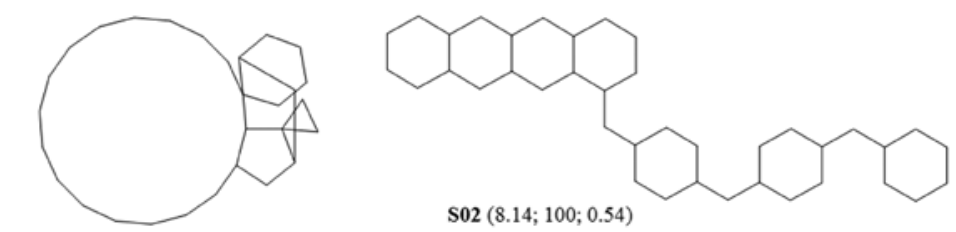

S01 $(8.29 ; 100 ; 0.95)$

S02 $(8.14 ; 100 ; 0.54)$

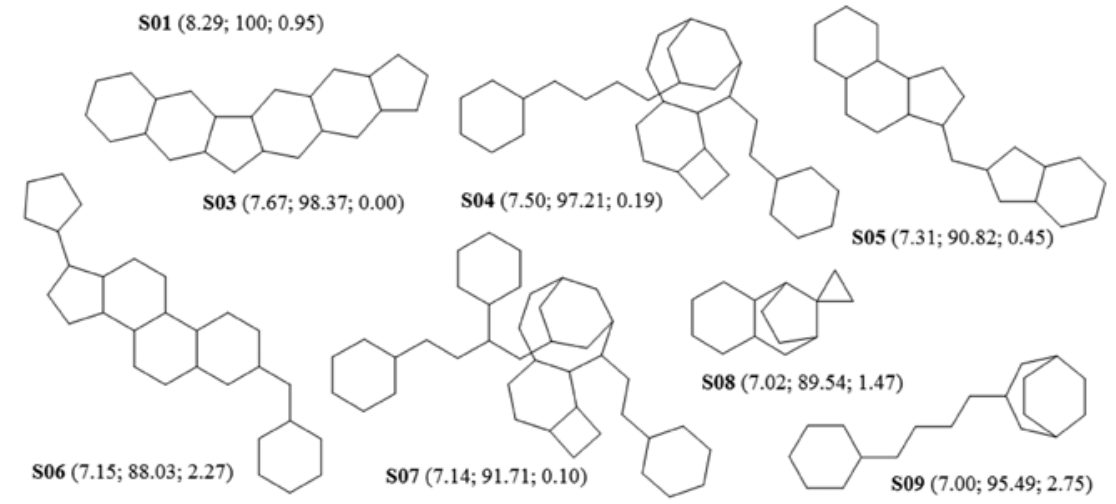

Figure 2. The Bemis-Murcko skeletons with the optimal performance are presented with the $\mathrm{A}_{\mathrm{pGI}}, \mathrm{P}_{\mathrm{pGI}}$ and $\mathrm{O}_{\mathrm{pGI}}$ scores (illustrated in the figure in order of presentation from left to right). S01-S09 represent each scaffold number. $\mathrm{A}_{\mathrm{pGI}}$, average of pGI values; $\mathrm{P}_{\mathrm{pGI}}$, number of pGI values the $>5$ value threshold; $\mathrm{O}_{\mathrm{pGI}}$, number of pGI upper boundary outliers.

The analysis of the anti-proliferative potential of each plain ring was performed firstly at a global level, taking into account all occurrences of a certain ring in the analyzed data set. This method ignores the synergistic or antagonistic effect produced by the presence in the same molecule of other rings. The top most frequently used scaffolds (A01-A20) are presented in Table II along with their frequency (in descending order) and corresponding global scores $\mathrm{A}_{1 \mathrm{D}}, \mathrm{A}_{\mathrm{pGI}}, \mathrm{P}_{1 \mathrm{D}}, \mathrm{P}_{\mathrm{pGI}}$, $\mathrm{O}_{\mathrm{ID}}$, and $\mathrm{O}_{\mathrm{pGI}}$.

The analysis of the $\mathrm{A}_{1 \mathrm{D}}$ and $\mathrm{A}_{\mathrm{pGI}}$ scores indicated that of these top favorite rings, the optimal anti-proliferative effects were produced by quinoline (A08), tetrahydropyran (A05), benzimidazole (A11) and pyrazole (A14). The same 4 rings exhibited the optimal $\mathrm{P}_{1 \mathrm{D}}$ and $\mathrm{P}_{\mathrm{pGI}}$ scores, even if the ranking order differed slightly. The outlier type scores, $\mathrm{O}_{1 \mathrm{D}}$ and $\mathrm{O}_{\mathrm{pGI}}$, exhibited similar values for all 20 rings. The importance of these rings can be also observed by comparing their scores with the corresponding Bemis-Murcko structures, such as BM05 in the case of pyrazole, BM02 for quinoline, BM04 for benzimidazole and BM01 for tetrahydropyran, highlighting the weight of the heteroatoms nature over the general topology.

The present study subsequently focused on the PGI set, as the compounds in this set have a higher potency. The analysis returned 8,725 distinct rings. The scores were analyzed if the total number of data points used was $>500$, yielding 685 rings.

The structural analysis focused on the 40 rings (B01-B40) that had both $\mathrm{A}_{\mathrm{pGI}}$ values $>6$ and $\mathrm{P}_{\mathrm{pGI}}>60$. The majority of these rings have complex structures and originate from natural compounds with well-established anticancer properties. Some representative ring structures are presented in Fig. 3 together with their $\mathrm{A}_{\mathrm{pGI}}, \mathrm{P}_{\mathrm{pGI}}$, and $\mathrm{O}_{\mathrm{pGI}}$ scores and with examples of well-known drugs that feature them.

Plain ring independent analysis. The analysis of the global effect of each ring structure ignored that the anti-proliferative effect can be influenced by the presence in the molecular structure of other rings. In order to better evaluate the contribution of each ring structure, the analysis was performed only on the 30,577 compounds of the AL set that contain only one ring in their structure. The majority of the A01-A20 rings presented lower performance scores in the independent analysis when compared with the global approach. The most significant difference was observed in the case of the quinoxaline structure (A18), which has a $\mathrm{P}_{\mathrm{pGI}}$ value of 39.94 and an $\mathrm{A}_{\mathrm{pGI}}$ value of 4.89 in compounds that have only one ring vs. a $\mathrm{P}_{\mathrm{pGI}}$ value of 15.90 and an $\mathrm{A}_{\mathrm{pGI}}$ value of 4.48 in all the compounds. These results indicate that the association of the quinoxaline ring with other rings lowers the anticancer potential.

Analyzing the optimal $\mathrm{P}_{\mathrm{pGI}}$ and $\mathrm{A}_{\mathrm{pGI}}$ scores for the independent effect of each ring for which the 500 data point rule was respected, yielded as the optimal results, the structures presented in Fig. 3. It can be observed that these 7 rings presented in Fig. 4 belong to the B01-B40 set, but not all B01-B40 rings exhibited significant score values in the independent analysis.

The most interesting rings appear to be B11, B13 and B20. The ring B13 is the skeleton of colchicine, a tricyclic alkaloid extracted from the plant Colchicum autumnale that strongly inhibits cellular mitosis by binding tubulin (30), while B11 is a bioisosteric derivative of $\mathrm{B} 13$ found in the tubulin-binding allocolchicine (31). Based on the $\mathrm{O}_{\mathrm{pGI}}$ score, the 4,8-dihydrobenzodithiophene-4,8-dione structure (B20) may be used to develop novel potent, yet selective, anti-proliferative drugs.

\section{Discussion}

The scope of present study was to perform an objective analysis of the impact of ring structures on the anti-proliferative effects in drug design. The results indicate that medicinal chemistry specialists focus their research on simple scaffolds, even if most of these have no significant pay-off guarantees. The reason is probably that the optimal chemical structures are complex and difficult to synthesize, and are therefore associated with high research costs. The use of a simple scaf- 
Table II. Most commonly used scaffolds and their corresponding global scores.

\begin{tabular}{|c|c|c|c|c|c|c|c|c|}
\hline Code & Scaffold & Freq (\%) & $A_{1 D}$ & $A_{p G i}$ & $P_{1 D}$ & $\mathrm{P}_{\mathrm{PGI}}$ & $\mathrm{O}_{1 \mathrm{D}}$ & $\mathrm{O}_{\mathrm{pGI}}$ \\
\hline $\mathrm{A} 01$ & & 45.51 & 88.19 & 4.66 & 9.93 & 25.98 & 2.66 & 4.29 \\
\hline $\mathrm{A} 02$ & & 3.19 & 87.48 & 4.79 & 11.68 & 34.03 & 2.70 & 3.97 \\
\hline $\mathrm{A} 03$ & & 1.58 & 89.47 & 4.62 & 9.91 & 25.33 & 2.92 & 4.11 \\
\hline $\mathrm{A} 04$ & & 1.36 & 89.87 & 4.68 & 8.47 & 27.58 & 2.93 & 4.64 \\
\hline $\mathrm{A} 05$ & & 1.20 & 69.12 & 4.98 & 24.90 & 36.69 & 2.23 & 3.28 \\
\hline A06 & & 1.04 & 83.54 & 4.83 & 12.77 & 34.40 & 3.20 & 4.12 \\
\hline $\mathrm{A} 07$ & & 0.96 & 86.63 & 4.79 & 10.75 & 33.34 & 2.67 & 3.91 \\
\hline $\mathrm{A} 08$ & & 0.92 & 77.76 & 5.02 & 17.57 & 46.34 & 2.96 & 3.95 \\
\hline $\mathrm{A} 09$ & & 0.91 & 87.68 & 4.73 & 10.27 & 31.31 & 2.48 & 3.90 \\
\hline A 10 & & 0.88 & 85.69 & 4.58 & 10.92 & 21.20 & 2.74 & 4.89 \\
\hline A11 & & 0.74 & 80.24 & 4.85 & 15.03 & 35.37 & 2.63 & 3.83 \\
\hline
\end{tabular}

fold is based on the low-risk approach and is very similar to the repurposing strategy (32). However, the present study has some limitations, considering the use of only one chemical repository. Anticancer research is far more complex and the NCI data represent just one sample.
Drug repurposing or drug repositioning is the identification of novel therapeutic uses for known drugs as an alternative to the long and expensive drug development programs beginning from scratch (33-35). It is considered that the same thinking paradigm is used for heterocyclic 
Table II. Continued.

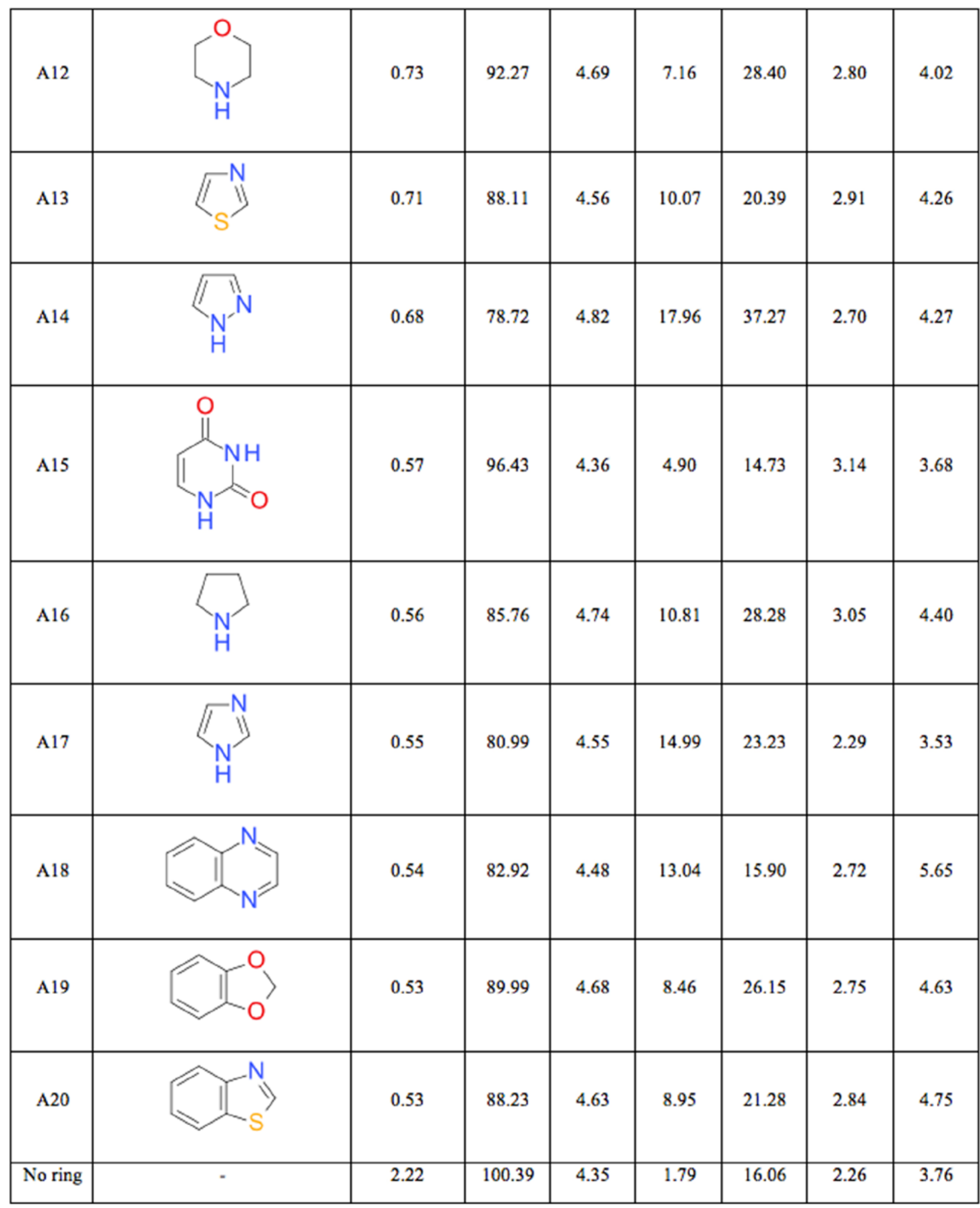

$\mathrm{A}_{1 \mathrm{D}}$, average of GI\% values; $\mathrm{P}_{1 \mathrm{D}}$, number of GI\% values the $<50 \%$ value threshold; $\mathrm{O}_{1 \mathrm{D}}$, number of GI\% lower boundary outliers; $\mathrm{A}_{\mathrm{pGI}}$, average of pGI values; $\mathrm{P}_{\mathrm{pGI}}$, number of $\mathrm{pGI}$ values the $>5$ value threshold; $\mathrm{O}_{\mathrm{pGI}}$, number of $\mathrm{pGI}$ upper boundary outliers.

rings. The repurposing of chemical scaffolds is based on the privileged structure concept. These types of structures are chemically accessible, have flexible and potent binding affinities towards a large type of biological targets, and possess good drug-like properties (36).
If the privileged structure concept is focused on the assessment of the potential for a particular ring structure to interact with a number of targets, the present study was centered on the objective evaluation of the anti-proliferative potential of cyclic structures as scaffolds for anticancer drug design, regardless 


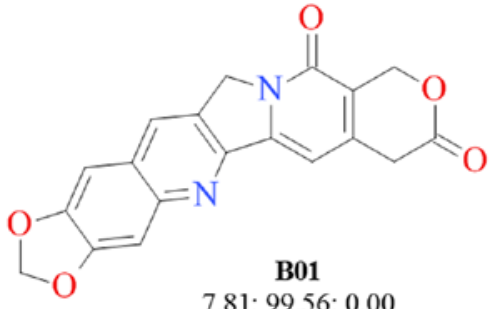

7.81:99.56:0.00

10,11-Methylenedioxy-20S-camptothecin

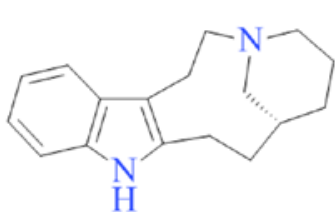

B07

$7.09 ; 81.41 ; 0.94$

Vincristine<smiles>O=c1ccc2nc3ccccc3oc-2c1</smiles>

B08

$7.08 ; 90.14 ; 1.44$

Dactinomycin

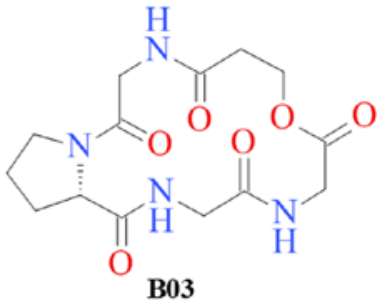

7.34; $93.88 ; 1.31$

Dactinomycin

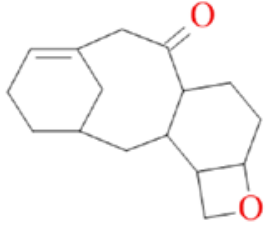

B06

$7.11 ; 89.32 ; 0.2$ Paclitaxel

Figure 3. The ring structures with the optimal performance presented with the $\mathrm{A}_{\mathrm{pGI}}, \mathrm{P}_{\mathrm{pGI}}$ and $\mathrm{O}_{\mathrm{pGI}}$ (illustrated in the figure in order of presentation from left to right) scores and examples of well-known drugs containing them. B01-B09 represent the number of each ring. $\mathrm{A}_{\mathrm{pGI}}$, average of $\mathrm{pGI}$ values; $\mathrm{P}_{\mathrm{pGI}}$, number of $\mathrm{pGI}$ values the $>5$ value threshold; $\mathrm{O}_{\mathrm{pGI}}$, number of pGI upper boundary outliers.<smiles>O=C1Cc2cc3n(c(=O)c2CO1)Cc1cc2cc4c(cc2nc1-3)OCO4</smiles>

$7.81 ; 99.60 ; 0.00$<smiles>c1ccc2c(c1)CCCc1ccccc1-2</smiles>

B11

$6.82 ; 85.03 ; 1.20$<smiles>O=C1Cc2cc3n(c(=O)c2CO1)Cc1cc2ccccc2nc1-3</smiles>

B17
$6.57 ; 86.87 ; 0.21$<smiles>CCCCC(C)C</smiles>

$6.92 ; 99.50 ; 1.31$<smiles>O=c1[nH]c2ccccc2n2cc3ccccc3c12</smiles>

B19

$6.50 ; 85.00 ; 1.10$<smiles>O=c1cccc2c(c1)CCCc1ccccc1-2</smiles>

B13 $6.87 ; 87.47 ; 0.43$

B20

$6.34 ; 98.32 ; 5.54$

Figure 4. The optimal 7 rings based on their $\mathrm{A}_{\mathrm{pGI}}, \mathrm{P}_{\mathrm{pGI}}$ and $\mathrm{O}_{\mathrm{pG}} \mathrm{I}$ scores (illustrated in the figure in order of presentation from left to right) calculated as independent effects. B01-B19 represent the number of each ring. $\mathrm{A}_{\mathrm{pGI}}$, average of $\mathrm{pGI}$ values; $\mathrm{P}_{\mathrm{pGI}}$, number of $\mathrm{pGI}$ values the $>5$ value threshold; $\mathrm{O}_{\mathrm{pGI}}$, number of pGI upper boundary outliers.

of their mechanisms of action. The scoring method in the present study provides an important new tool that could be successfully combined with the privileged structure analysis methods to identify better scaffolds. For example, the quinoline ring (A08) emerged in the present study as an effective ring and has been demonstrated as a privileged scaffold in cancer drug development $(37,38)$. The pyrazole ring (A14) is another privileged structure, particularly when targeting protein kinases $(39,40)$, which can generate interesting leads providing that it is joined by other heterocyclic structures.

The present study used a dual strategy, a topological method using Bemis-Murcko scaffolds to assess the importance of rings interconnection networks (39), and a plain ring analysis to reveal the importance of specific heteroatoms and their relative position. In some cases, the results of both methods were similar. One important example is in the case of taxene derivatives. Paclitaxel is represented by the Bemis-Murcko scaffold S07 and by the plain ring B06, both with $A_{p G I}$ scores $>7$. Docetaxel, a congener of paclitaxel, shares the ring B06, but is represented by the more potent Bemis-Murcko scaffold S04. Another important observation is the practice of structural simplification, a method of molecular truncation used to avoid large and low drug-like scaffolds (41).

In conclusion, the scoring method developed and implemented herein can easily be used in any other similar research based on the NSC data. The method could focus on a particular cyclic structure and it can be used to find other ring structures with a similar inhibition profile. Even if the method used in the present study ignored the effects of substituents on the cyclic structures, the results clearly 
indicate that the nature of the ring structure can have a significant impact on the anticancer potential. Likewise, this method can be used in future research to quantify the impact of the ring structure compared to that of the substituents nature or position.

\section{Acknowledgements}

Not applicable.

\section{Funding}

No funding was received.

\section{Availability of data and materials}

Data sharing is not applicable to this article, as no datasets were generated or analyzed during the current study.

\section{Authors' contributions}

GMN was involved in the conceptualization of the study. All authors (GNDI, OTO, GN, IIO, AT, TIB, DAS and GMD) were involved in the study methodology. All authors have read and approved the final manuscript.

\section{Ethics approval and consent to participate}

Not applicable.

\section{Patient consent for publication}

Not applicable.

\section{Competing interests}

DAS is the Editor-in-Chief for the journal, but had no personal involvement in the reviewing process, or any influence in terms of adjudicating on the final decision, for this article. All the other authors declare that they have no competing interests.

\section{References}

1. Schirrmacher V: From chemotherapy to biological therapy: A review of novel concepts to reduce the side effects of systemic cancer treatment (Review). Int J Oncol 54: 407-419, 2019.

2. Ferreira LG, Dos Santos RN, Oliva G and Andricopulo AD: Molecular docking and structure-based drug design strategies. Molecules 20: 13384-13421, 2015.

3. Śledź P and Caflisch A: Protein structure-based drug design: From docking to molecular dynamics. Curr Opin Struct Biol 48 93-102, 2018

4. Lionta E, Spyrou G, Vassilatis DK and Cournia Z: Structure-based virtual screening for drug discovery: Principles, applications and recent advances. Curr Top Med Chem 14: 1923-1938, 2014.

5. Chen KC, Juang SH and Lien JC: Identification of antiproliferative emodin analogues as inhibitors of epidermal growth factor receptor in cancer. Int J Mol Med 43: 1281-1288, 2019.

6. Martin YC, Kofron JL and Traphagen LM: Do structurally similar molecules have similar biological activity? J Med Chem 45: 4350-4358, 2002

7. Sun H, Tawa G and Wallqvist A: Classification of scaffold-hopping approaches. Drug Discov Today 17: 310-324, 2012.

8. Gomez L: Decision Making in Medicinal Chemistry: The Power of Our Intuition. ACS Med Chem Lett 9: 956-958, 2018.
9. Segall $\mathrm{M}$ and Chadwick A: The risks of subconscious biases in drug-discovery decision making. Future Med Chem 3: 771-774, 2011.

10. Sharma PC, Bansal KK, Sharma A, Sharma D and Deep A: Thiazole-containing compounds as therapeutic targets for cancer therapy. Eur J Med Chem 188: 112016, 2020.

11. Dadashpour S and Emami S: Indole in the target-based design of anticancer agents: A versatile scaffold with diverse mechanisms. Eur J Med Chem 150: 9-29, 2018.

12. Mazumder A, Cerella C and Diederich M: Natural scaffolds in anticancer therapy and precision medicine. Biotechnol Adv 36: $1563-1585,2018$.

13. Munteanu AC, Badea M, Olar R, Silvestro L, Dulea C, Negut CD and Uivarosi V: Synthesis and Structural Investigation of New Bio-Relevant Complexes of Lanthanides with 5-Hydroxyflavone: DNA Binding and Protein Interaction Studies. Molecules 21: 1737, 2016.

14. Ismail NSM, Ali EMH, Ibrahim DA, Serya RAT and Abou El Ella DA: Pyrazolo[3,4-d]pyrimidine based scaffold derivatives targeting kinases as anticancer agents. Futur J Pharm Sci 2: 20-30, 2016.

15. Nitulescu GM, Matei L, Aldea IM, Draghici C, Olaru OT and Bleotu C: Ultrasound-assisted synthesis and anticancer evaluation of new pyrazole derivatives as cell cycle inhibitors. Arab J Chem 12: 816-824, 2019.

16. Nitulescu GM, Draghici C, Olaru OT, Matei L, Ioana A, Dragu LD and Bleotu C: Synthesis and apoptotic activity of new pyrazole derivatives in cancer cell lines. Bioorg Med Chem 23: 5799-5808, 2015.

17. Nitulescu GM, Draghici C and Olaru OT: New potential antitumor pyrazole derivatives: Synthesis and cytotoxic evaluation. Int J Mol Sci 14: 21805-21818, 2013.

18. Nitulescu GM, Paunescu H, Draghici C, Missir AV, Coman OA and Fulga I: Synthesis and pharmacological evaluation of some new pyrazole derivatives. Farmacia 58: 190-197, 2010.

19. Takimoto CH: Anticancer drug development at the US National Cancer Institute. Cancer Chemother Pharmacol 52 (Suppl 1): S29-S33, 2003

20. DeVita VT Jr and Chu E: A history of cancer chemotherapy. Cancer Res 68: 8643-8653, 2008.

21. Kim HS, Sung YJ and Paik S: Cancer Cell Line Panels Empower Genomics-Based Discovery of Precision Cancer Medicine. Yonsei Med J 56: 1186-1198, 2015.

22. Shoemaker RH: The NCI60 human tumour cell line anticancer drug screen. Nat Rev Cancer 6: 813-823, 2006.

23. Zaharevitz DW, Holbeck SL, Bowerman C and Svetlik PA: COMPARE: A web accessible tool for investigating mechanisms of cell growth inhibition. J Mol Graph Model 20: 297-303, 2002.

24. Fang X, Shao L, Zhang H and Wang S: Web-based tools for mining the NCI databases for anticancer drug discovery. J Chem Inf Comput Sci 44: 249-257, 2004.

25. Nitulescu GM, Soriga SG, Socea LI, Olaru OT and Plesu V: Structure-activity relationships and chemoinformatic analysis of the anticancer profile of an aminopyrazole derivative. Rev Chim 67: 162-165, 2016

26. Nitulescu GM, Iancu G, Nitulescu G, Iancu RC, Bogdanici C and Vasile D: Brave new hope for breast cancer: Aminopyrazole derivates between rational design and clinical efficacy. Rev Chim 68: 754-757, 2017.

27. Herbowski L: Skeletal muscle metastases from papillary and follicular thyroid carcinomas: An extensive review of the literature. Oncol Lett 15: 7083-7089, 2018.

28. Lipkus AH, Yuan Q, Lucas KA, Funk SA, Bartelt WF III, Schenck RJ and Trippe AJ; CAS Registry: Structural diversity of organic chemistry. A scaffold analysis of the CAS Registry. J Org Chem 73: 4443-4451, 2008.

29. Néda Z, Varga L and Biró TS: Science and Facebook: The same popularity law! PLoS One 12: e0179656, 2017.

30. Ahmed K, Zaidi SF, Cui Z-G, Zhou D, Saeed SA and Inadera H Potential proapoptotic phytochemical agents for the treatment and prevention of colorectal cancer. Oncol Lett 18: 487-498, 2019.

31. Paymode DJ andRamana CV: Total Synthesis of ( \pm )-Allocolchicine and Its Analogues Using Co-Catalyzed Alkyne $[2+2+$ 2]-Cyclotrimerization. ACS Omega 2: 5591-5600, 2017.

32. Armando RG, Mengual Gómez DL and Gomez DE: New drugs are not enough drug repositioning in oncology: An update. Int $\mathbf{J}$ Oncol 56: 651-684, 2020.

33. Pushpakom S, Iorio F, Eyers PA, Escott KJ, Hopper S, Wells A, Doig A, Guilliams T, Latimer J, McNamee C, et al: Drug repurposing: Progress, challenges and recommendations. Nat Rev Drug Discov 18: 41-58, 2019. 
34. Ion GND, Mihai DP, Lupascu G and Nitulescu GM: Application of molecular framework-based data-mining method in the search for beta-secretase 1 inhibitors through drug repurposing. J Biomol Struct Dyn 37: 3674-3685, 2019.

35. Yang X, Huang W-T, Wu H-Y, He R-Q, Ma J, Liu A-G and Chen G: Novel drug candidate for the treatment of several soft tissue sarcoma histologic subtypes: A computational method using survival associated gene signatures for drug repurposing. Oncol Rep 41: 2241-2253, 2019.

36. Schneider P and Schneider G: Privileged Structures Revisited. Angew Chem Int Ed Engl 56: 7971-7974, 2017.

37. Musiol R: An overview of quinoline as a privileged scaffold in cancer drug discovery. Expert Opin Drug Discov 12: 583-597, 2017.

38. Afzal O, Kumar S, Haider MR, Ali MR, Kumar R, Jaggi M and Bawa S: A review on anticancer potential of bioactive heterocycle quinoline. Eur J Med Chem 97: 871-910, 2015.
39. Cui YJ, Tang LQ, Zhang CM and Liu ZP: Synthesis of Novel Pyrazole Derivatives and Their Tumor Cell Growth Inhibitory Activity. Molecules 24: 279, 2019.

40. Nitulescu GM, Nedelcu G, Buzescu A and Olaru OT: Aminopyrazoles as privileged structures in anticancer drug design - an in silico study. Izv Him 48: 55-60, 2016.

41. Wang S, Dong G and Sheng C: Structural simplification: An efficient strategy in lead optimization. Acta Pharm Sin B 9: 880-901, 2019.

(i) $\Theta$ This work is licensed under a Creative Commons Attribution-NonCommercial-NoDerivatives 4.0 International (CC BY-NC-ND 4.0) License. 